Bioscientia Medicina: Journal of Biomedicine \& Translational Research

Journal Homepage: www.bioscmed.com

\title{
Laparoscopic Davydov Procedure for Creation of Neovagina in MRKH Syndrome
}

\section{Ratih Krisna $^{1 *}$, Rizky Agustria ${ }^{1}$}

${ }^{1}$ Obstetric and Gynecology Department Urogynecology Division Moh. Hoesin Hospital, Palembang, Indonesia

\author{
A R T I C L E I N F O \\ Keywords: \\ MRKH Syndrome, \\ Davydov Procedure, \\ Primary Amenorrhea, \\ Vaginoplasty
}

\section{*Corresponding author: \\ Ratih Krisna \\ E-mail address: \\ ratihkrisna@yahoo.co.id}

All authors have reviewed and approved the final version of the manuscript.

https://doi.org/10.32539/bsm.v5i9.348

\begin{abstract}
A B S T R A C T
Background: The Mayer-Rokitansky-Kuster-Hauser (MRKH) syndrome is a condition in which there is an absence of the uterus and the upper part $(2 / 3)$ of the vagina. Women with MRKH syndrome show normal development of secondary sexual characteristics and a normal 46, XX karyotype. MRKH syndrome is a common cause of primary amenorrhea. Ultrasonography examination confirms kidneys, presence of the ovaries, and absence of uterus. Treatment should include a multidisciplinary approach to create a functional vagina. One of the procedures is laparoscopic Davydov vaginoplasty. Aim : To report MRKH syndrome case treated with laparoscopic Davydov vaginoplasty. Method : Ms. A, 21 years old, POA0, came to FER clinic Moh. Hoesin Hospital Palembang with complaint, never had menstruation, the patient admitted her breast, and her pubic had growth. She wants to get married in the next six months. From the US result, there was non visualized uterus, genital band $(+)$, and both ovaries within normal limit $\sim \mathrm{MRKH}$ Syndrome. Karyotype Examination: 46, XX. Patient was diagnosed with primary amenorrhea caused by MRKH syndrome and was planned for neovagina (Davydov Procedure). Result : A woman 21-year-old, POA0, was diagnosed with primary amenorrhea caused by MRKH syndrome. The Davydov procedure was done to create a neovagina. Intraoperative, after protrusion of the wood mold as a marker shown, an incision is made to the left and right side so that the mold penetrates the abdominal cavity, then the mold is pulled into the vaginal canal slowly. The anterior and posterior peritoneal layers are drawn into the vaginal canal. The anterior and posterior peritoneal layers were withdrawn with an ovum clamp and then interrupted suture. The anterior and posterior peritoneal layers were sutured in a circular method to form a vaginal cuff. The vaginal wall is sutured (interrupted) to hold mold fixed. Conclusion : MRKH syndrome is a disorder that occurs in females and mainly affects the reproductive system. This condition causes the vagina and the uterus to be underdeveloped or even absent at birth. Patients with diagnosed MRKH syndrome require interdisciplinary care. Davydov procedure is a laparoscopically-assisted technique, which has an advantage over traditional approaches. It has shorter operating time, lower intraoperative complications, shorter hospital stay, and no external scars. Postoperatively, sexual function is similar to women without gynecological disorders.
\end{abstract}

\section{Introduction}

The Mayer-Rokitansky-Kuster-Hauser

(MRKH) syndrome is a condition in which there is an absence of the uterus and the upper part $(2 / 3)$ of the vagina. Women with MRKH syndrome show normal development of secondary sexual characteristics and a normal 46, XX karyotype. MRKH syndrome is a common cause of primary amenorrhea. Isolated uterovaginal aplasia is called Rokitansky sequence or to type I (isolated) MRKH syndrome. ${ }^{1}$ Incomplete aplasia or associated with other malformations is called MURCS association (or type II MRKH syndrome). The first clinical signal is primary amenorrhea in patients presenting with a normal female phenotype, and normal functioning ovaries with no sign of androgen excess. External examination reveals completed puberty with normal secondary female sexual characteristics (pubic hair and breast development are Tanner stage 5) and normal external genitalia. At the same time, the vagina is reduced to a more or less deep 
$(2-7 \quad \mathrm{~cm})$ vaginal dimple. ${ }^{2}$ Transabdominal ultrasonograph is a simple and noninvasive method, and must be the first investigation in evaluating patients with suspected Mullerian aplasia. Ultrasonography examination confirms kidneys, presence of the ovaries, and absence of uterus. MRI is a non-invasive technique that provides a more sensitive and more specific means of diagnosis than ultrasonography. It should be performed when ultrasonographic findings are inconclusive or incomplete, since failure to clearly identify the uterus or Mullerian rudiments or ovaries does not necessarily imply their absence. MRI allows an accurate evaluation of the uterine aplasia, as well as a clear visualization of the rudimentary horns and ovaries. ${ }^{1,2}$

Differential diagnosis of Mullerian aplasia includes patients presenting with primary amenorrhea and with normal secondary sexual characteristics. This should first lead to exclusion of gonadal dysgenesis. The differential diagnosis includes congenital absence of uterus and vagina (aplasia or agenesis), isolated vaginal atresia and androgen insensitivity. Ultrasonography can be used to define the Mullerian structures in infrequent cases where palpation is unrevealing. ${ }^{1-3}$

Treatment should include a multidisciplinary approach. Young women diagnosed with $\mathrm{MRKH}$ syndrome suffer from anxiety and psychological distress when told they have no uterus and vagina. Treatment of creating neovagina must be offered to patients only when they are ready to start sexual activity and also when they are emotionally mature. Treatment may be either surgical or nonsurgical. One of the surgical procedure to create a functional vagina is laparoscopic Davydov vaginoplasty. ${ }^{1-2}$

\section{Aim}

To report MRKH syndrome case treated with laparoscopic Davydov vaginoplasty

\section{Method}

Mrs.A, 21 years old, POA0, came to FER clinic Moh. Hoesin Hospital Palembang with complaint, never had menstruation. History of blood spotting $(+)$, history of abdominal pain (-), history of abdominal enlargement (-). Patient admitted her breast and her pubic growth had growth. Patient went to OBGYN at Hermina Hospital, and was performed US gynecology, with the US result uterine non visualized, then the patient was referred to Moh. Hoesin Hospital. Physical examination finding : general condition : weight $91 \mathrm{~kg}$; Height 163 cm (BMI : $34.3 \mathrm{~kg} / \mathrm{m} 2$, overweight). Sensorium compos mentis, BP 120/70 mmHg, P 80x/minute, RR $24 x /$ minute.. Secondary examination, breast has growth, pubic hair has growth, armpit hair has growth, tanner score : 3 .

Ultrasonography examination showed non visualized uterus, genital band $(+)$. Both ovaries had normal shape and size, right ovary size 3,68 x 1,86 $\mathrm{cm}$ and left ovary size 3,68 x 2,21 cm. Liver and lien in normal limit, both renal in normal limit. Both uterine non visualized, genital band $(+)$. Ovaries in normal limit MRKH Syndrome. Laboratorium ecamination within normal limit. Karyotype Examination: 46, XX. Patient was diagnosed with primary amenorrhea caused by MRKH syndrome and was planned for neovagina (Davydov Procedure).

\section{Result}

A woman 21-year-old, POA0, was diagnosed with primary amenorrhea caused by MRKH syndrome. The Davydov procedure was done to create a neovagina. Intraoperative, after protrusion of the wood mold as a marker shown, an incision is made to the left and right side so that the mold penetrates the abdominal cavity, then the mold is pulled into the vaginal canal slowly. The anterior and posterior peritoneal layers are drawn into the vaginal canal. The anterior and posterior peritoneal layers were withdrawn with an ovum clamp and then interrupted suture. The anterior and posterior peritoneal layers were sutured in a circular method to form a vaginal cuff. The vaginal wall is sutured (interrupted) to hold mold fixed.

\section{Discussion}

MRKH syndrome is a disorder that occurs in 
females and mainly affects the reproductive system. This condition causes the vagina and the uterus to be underdeveloped or even absent at birth. Patients typically present with primary amenorrhea in adolescence with normal growth and development. Sexual intercourse and infertility are also problematic. For the diagnosis of MRKH syndrome, transabdominal ultrasonography is a useful and noninvasive technique. It must be the first investigation in evaluating patients suspected of having the syndrome. ${ }^{1,2}$

The etiology of this syndrome remains unclear; however, genetic involvement has been confirmed by several investigators, showing autosomal dominant inheritance. ${ }^{1,3}$

The most common treatment for this syndrome is the construction of a neovagina and follow-up infertility treatment, as needed. The aim of the creation of a functional neovagina is so that the patient can still have sexual intercourse. Among the several surgical techniques available, the laparoscopic Davydov procedure, in which the vesicorectal space is coated by the peritoneum, is by far the simplest, safest, and quickest operative method for this type of case. Intraoperative and postoperative complications are rare compared with other surgical techniques. Timing for the surgical creation of a neovagina is elective and depends on each individual patient and the circumstances of each case. ${ }^{1,2}$

The laparoscopic Davydov procedure has numerous advantages. First, the procedure is quick and has a low risk of complications. Second, it is excellent esthetically because there are no surgical scars. The one disadvantage of these cases is that the patients required intermittent postoperative dilation. ${ }^{1,3}$
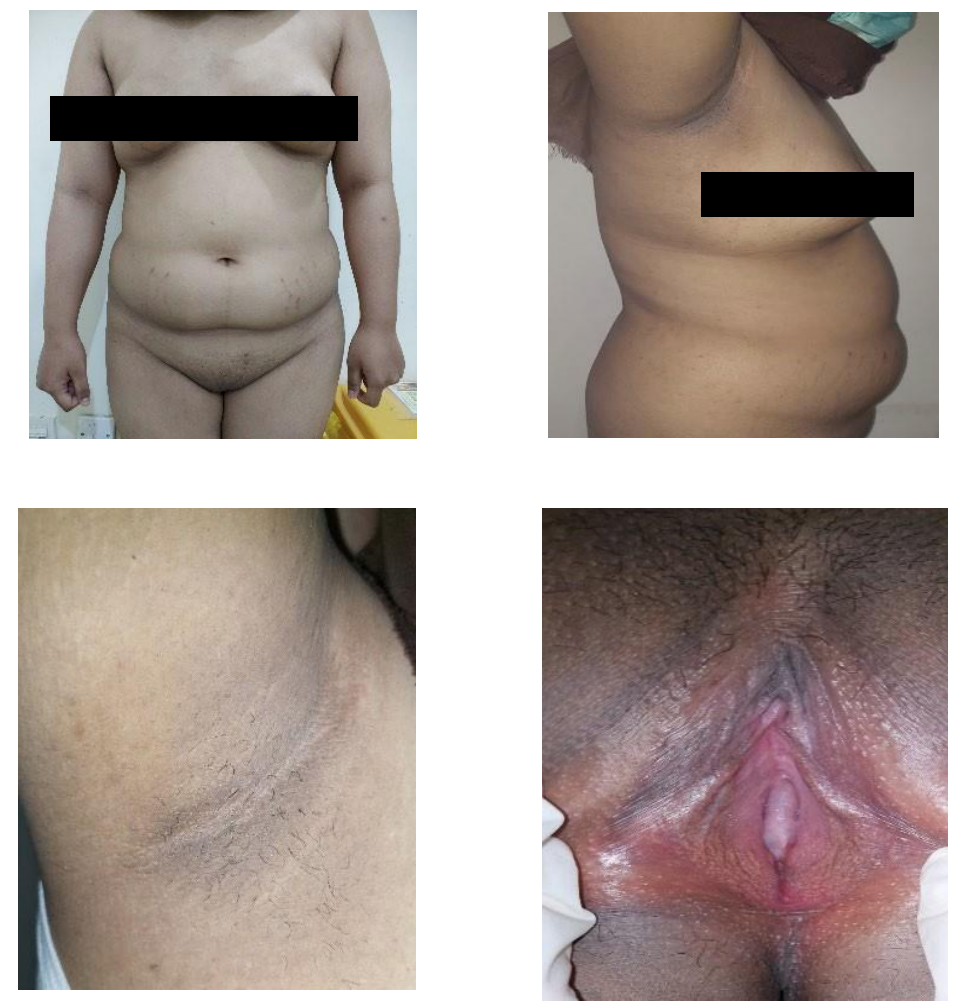

Figure 1. Secondary characteristic of Ms. A 

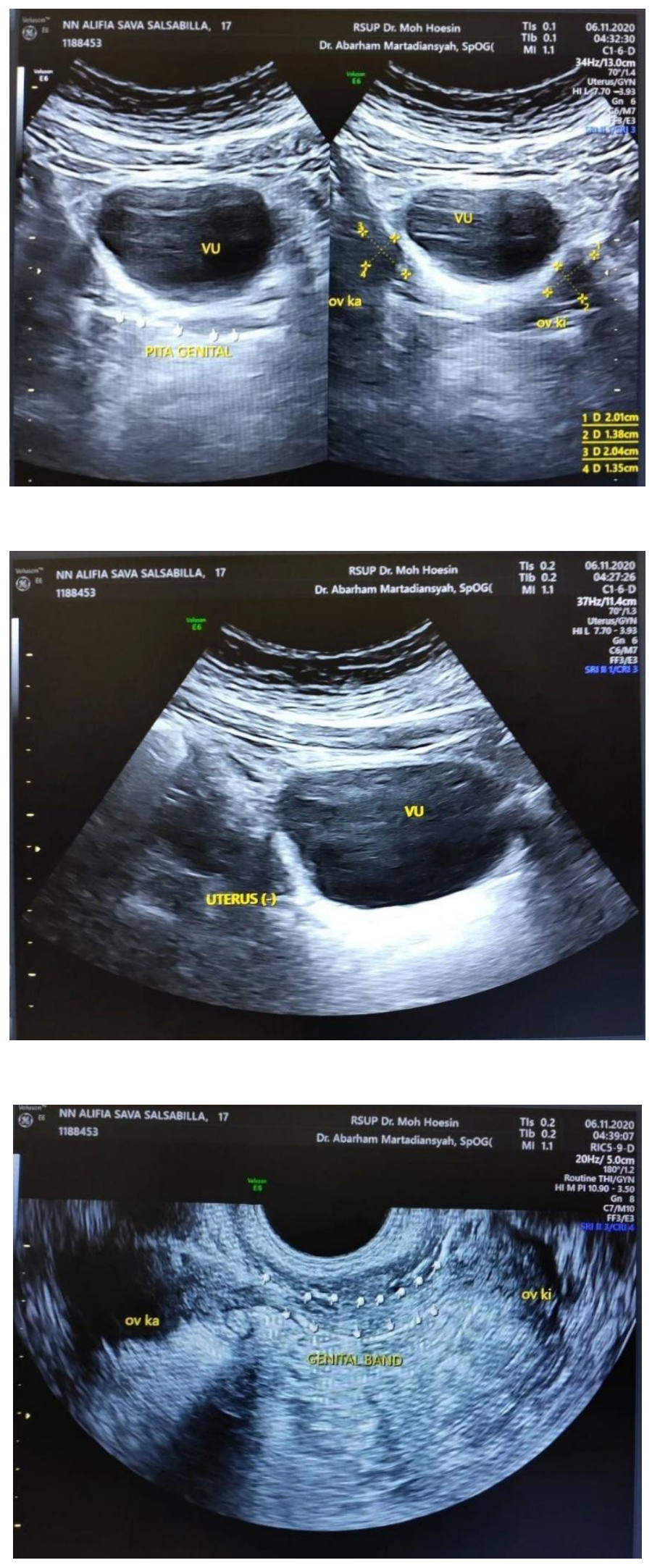

Figure 2. Ultrasonography finding of Ms. A 

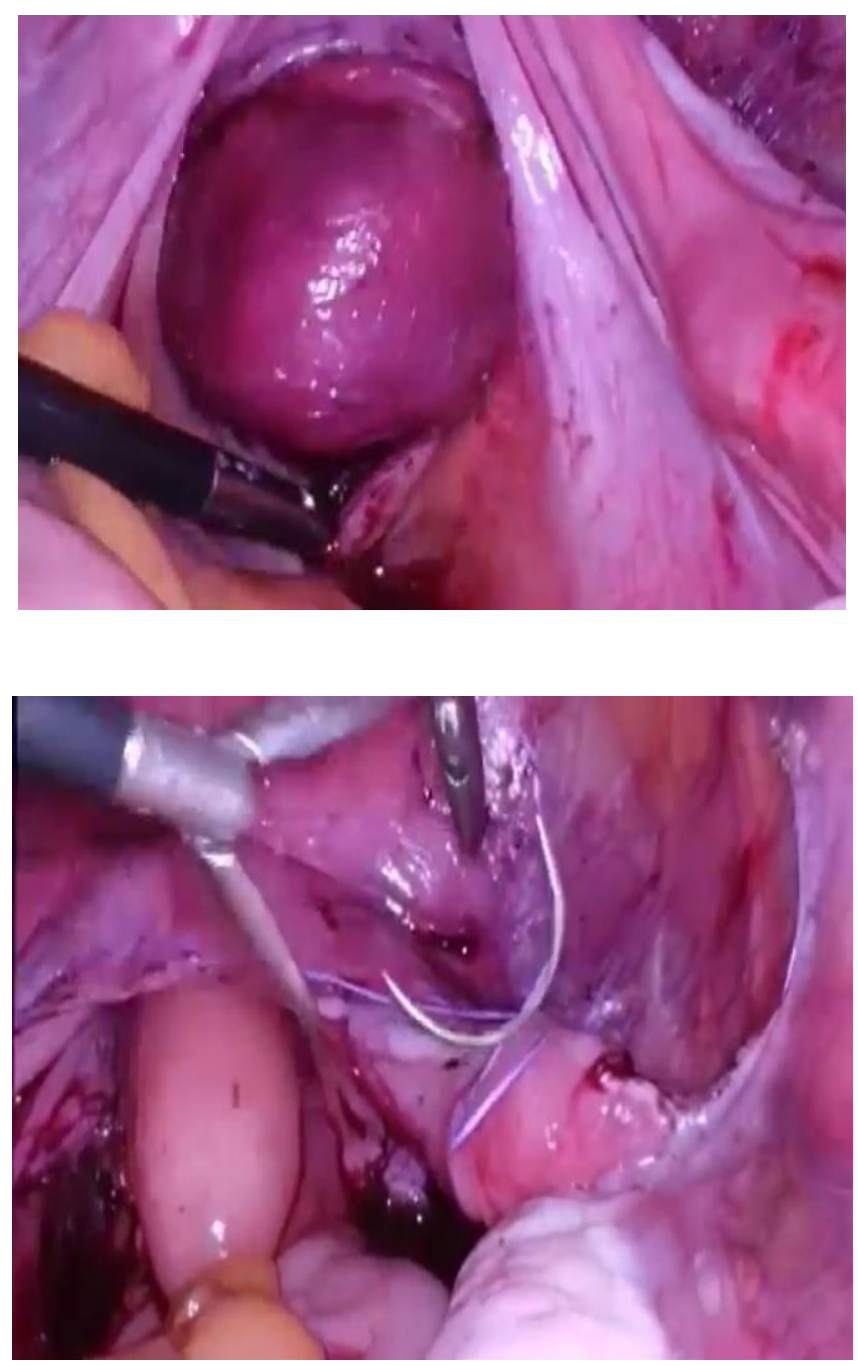

Figure 3. Intraoperative finding of Ms. A

\section{Conclusion}

MRKH syndrome is a disorder that occurs in females and mainly affects the reproductive system. This condition causes the vagina and the uterus to be underdeveloped or even absent at birth. Patients with diagnosed MRKH syndrome require interdisciplinary care. Davydov procedure is a laparoscopically-assisted technique, which has an advantage over traditional approaches. It has shorter operating time, lower intraoperative complications, shorter hospital stay, and no external scars. Postoperatively, sexual function is similar to women without gynecological disorders.
2. Bombard DS 2nd, Mousa SA. MayerRokitansky- Kuster-Hauser syndrome : complications, diagnosis and possible treatment options: a review. Gynecol Endocrinol 2014; 30: 618-23

3. Willemsen WN, Kluivers KB. Long-term results of vaginal construction with the use of peritoneal graft (Davydov procedure) in patients with Mayer- Rokitansky-Küster syndrome. Fertil Steril 2015; 103: 220

\section{References}

1. Londra L, Chuong FS, Kolp L. MayerRokitansky- Kuster-Hauser syndrome: a review. Int J Womens Health 2015; 7: 865-70. 